\title{
Evolutionary stasis of a deep subsurface microbial lineage
}

\author{
Eric D. Becraft ${ }^{1,2} \cdot$ Maggie C. Y. Lau Vetter $\mathbb{C}^{3,4} \cdot$ Oliver K. I. Bezuidt ${ }^{1} \cdot$ Julia M. Brown ${ }^{1} \cdot$ Jessica M. Labonté $\mathbb{C}^{5}$. \\ Kotryna Kauneckaite-Griguole ${ }^{6} \cdot$ Ruta Salkauskaite $^{6} \cdot$ Gediminas Alzbutas $^{6}$ - Joshua D. Sackett ${ }^{7}$. \\ Brittany R. Kruger ${ }^{7}$ Vitaly Kadnikov ${ }^{8} \cdot$ Esta van Heerden ${ }^{9,10} \cdot$ Duane Moser $^{7} \cdot$ Nikolai Ravin $^{8} \cdot$ Tullis Onstott $^{4}$. \\ Ramunas Stepanauskas $\mathbb{D}^{1}$
}

Received: 22 April 2020 / Revised: 26 February 2021 / Accepted: 15 March 2021 / Published online: 6 April 2021

(c) The Author(s) 2021. This article is published with open access

\begin{abstract}
Sulfate-reducing bacteria Candidatus Desulforudis audaxviator (CDA) were originally discovered in deep fracture fluids accessed via South African gold mines and have since been found in geographically widespread deep subsurface locations. In order to constrain models for subsurface microbial evolution, we compared CDA genomes from Africa, North America and Eurasia using single cell genomics. Unexpectedly, 126 partial single amplified genomes from the three continents, a complete genome from of an isolate from Eurasia, and metagenome-assembled genomes from Africa and Eurasia shared $>99.2 \%$ average nucleotide identity, low frequency of SNP's, and near-perfectly conserved prophages and CRISPRs. Our analyses reject sample crosscontamination, recent natural dispersal, and unusually strong purifying selection as likely explanations for these unexpected results. We therefore conclude that the analyzed CDA populations underwent only minimal evolution since their physical separation, potentially as far back as the breakup of Pangea between 165 and $55 \mathrm{Ma}$ ago. High-fidelity DNA replication and repair mechanisms are the most plausible explanation for the highly conserved genome of CDA. CDA presents a stark contrast to the current model organisms in microbial evolutionary studies, which often develop adaptive traits over far shorter periods of time.
\end{abstract}

\section{Introduction}

Knowledge of mechanisms, rates and consequences of microbial evolution is critical to a wide range of scientific and practical endeavors, such as prevention and treatment of human diseases, environmental bioremediation, studies of global biogeochemical cycles and understanding the diversity of life. Our current concepts concerning microbial

Supplementary information The online version contains supplementary material available at https://doi.org/10.1038/s41396021-00965-3.

Ramunas Stepanauskas

rstepanauskas@bigelow.org

1 Bigelow Laboratory for Ocean Sciences, East Boothbay, ME, USA

2 Department of Biology, University of North Alabama, Florence, AL, USA

3 Institute of Deep-sea Science and Engineering, Chinese Academy of Sciences, Sanya, Hainan Province, P. R. China

4 Department of Geosciences, Princeton University, Princeton, NJ, USA evolution largely rely on genome-based inferences and experimental studies applied to a small number of fastproliferating species, primarily human pathogens and commensals [1-3]. Microbes in these laboratory-based evolutionary experiments inhabit energy- and nutrient-rich environments, have generation times ranging from minutes to days, and may accumulate nucleotide substitutions and acquire genes over the course of years or less. However, recent advances in environmental microbiology indicate that such microorganisms may be imperfect models for many bacteria and archaea, which may contain over a trillion

5 Department of Marine Biology, Texas A\&M University at Galveston, Galveston, TX, USA

6 Thermo Fisher Scientific Baltics, Vilnius, Lithuania

7 Division of Hydrologic Sciences, Desert Research Institute, Las Vegas, NV, USA

8 Institute of Bioengineering, Research Center of Biotechnology RAS, Moscow, Russia

9 Centre for Water Sciences and Management, North West University, Potchefstroom, South Africa

10 iWater, Bloemfontein, South Africa 
operationally-defined lineages [4-6] and pursue widely divergent life strategies that may affect evolutionary rates.

Essentially unacknowledged until 30 years ago, deep subsurface microorganisms are now estimated to constitute $\sim 10 \%$ of our planet's total biomass $[7,8]$ and are found to contain a large fraction of yet uncharacterized biological diversity, the so-called "microbial dark matter" [6, 9-11]. Many of these microorganisms rely upon low-yield energy sources, resulting in estimated generation times ranging from months to decades [12, 13]. The Firmicute Candidatus Desulforudis audaxviator MP104C (CDA), originally discovered in deep continental subsurface, has emerged as one of the model microorganisms for this environment [14-17]. The CDA metagenome-assembled genome (MAG) was based upon a near-clonal population of $\sim 10^{11}$ cells filtered from 5,600 liters of fracture water intersected at $2.8 \mathrm{~km}$ depth in the Mponeng gold mine in South Africa, where it appeared to form a single-species, chemoautotrophic ecosystem supported by $\mathrm{H}_{2}$, formate and sulfate that are generated in situ from radiolysis [14]. A subsequent analysis of five single amplified genomes (SAGs) of CDA relatives from $3 \mathrm{~km}$ deep fracture water in the neighboring Tau Tona gold mine identified novel prophages, retrons, CRISPRs, restriction-modification systems and transposases, which suggested that recombination, horizontal gene transfer (HGT) and viral infections played a significant role in the evolution of this lineage [18].

Here we report multiple SAGs of CDA from three continents: Africa (three subsurface boreholes accessed from the Mponeng, Beatrix and Tau Tona gold mines in the 3.0-2.8 Ga Witwatersrand Basin of South Africa); North America (borehole Inyo-BLM 1 (BLM1), accessing a regional fault-controlled, Paleozoic carbonate aquifer in the Death Valley Regional Flow System of southern Nevada and southeastern California); and Eurasia (borehole BY-1R, accessing a Cretaceous aquifer in the West Siberian artesian mega-basin). We also analyzed a second CDA MAG BY57 and a genome of the first laboratory culture, CDA BYF from the BY-1R site [15-17]. Given the large geographic distances separating the subsurface sampling sites, we hypothesized that CDA genomes should be genetically divergent. Further, because of the differences in the physicochemical conditions among the sampling sites, we also anticipated divergent adaptations to the local environments, i.e., that the evolutionary trajectories of the CDA populations would be analogous to those of Darwin's finches.

\section{Materials and methods}

\section{Field sample collection}

Deep fracture water was collected on January 21, 2011 from a borehole drilled at a depth of 1,339 $\mathrm{m}$ in Beatrix
Gold Mine, on October 6, 2014 from a borehole drilled at a depth of 3,402 $\mathrm{m}$ in Mponeng gold mine, and on January 19, 2012 from a borehole drilled at a depth of $3,316 \mathrm{~m}$ in Tau Tona gold mine (Fig. 1A). The methods used in collecting and analyzing samples from mine boreholes have been previously described [19]. Mponeng and

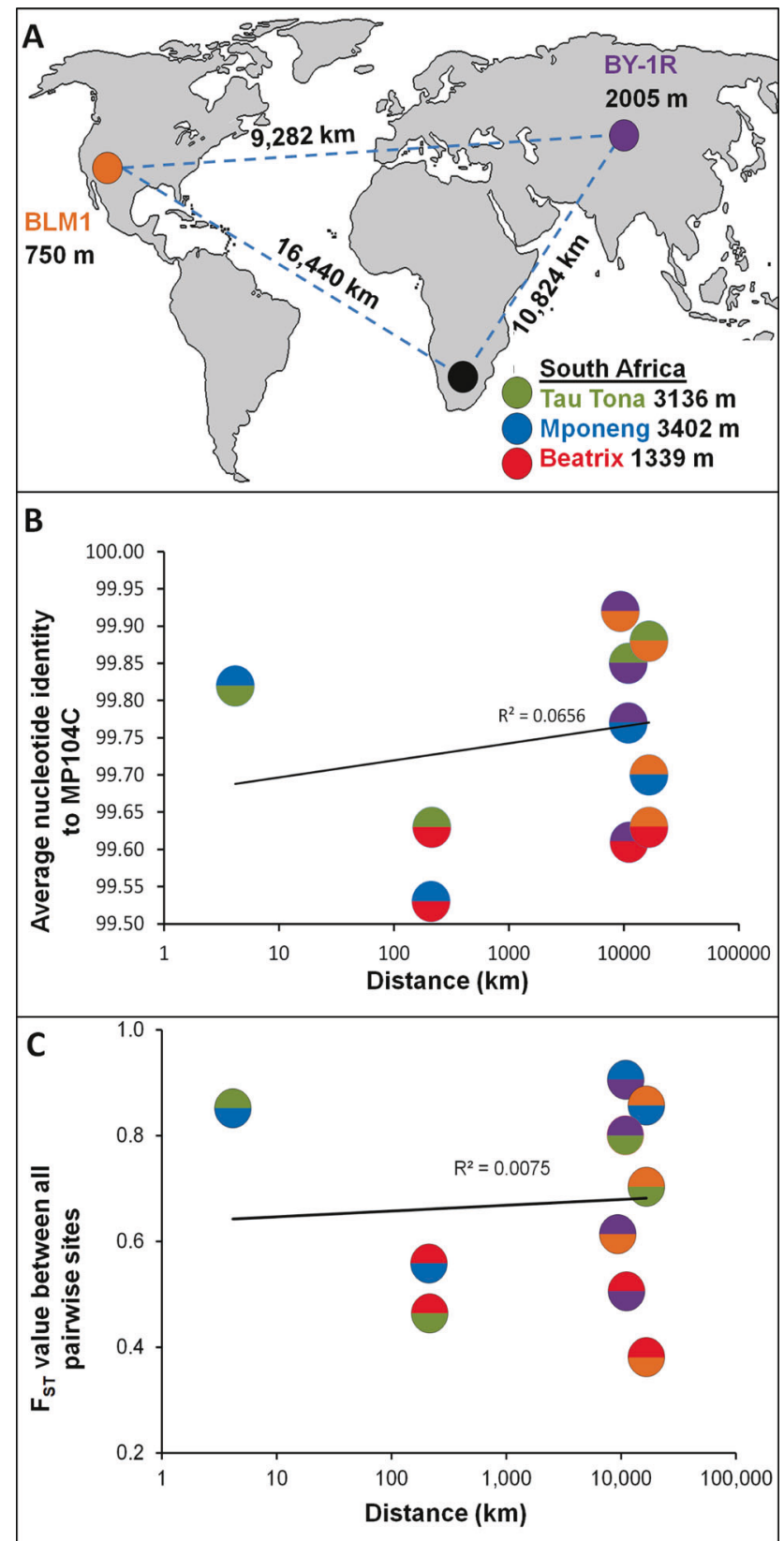

Fig. 1 General characteristics of the analyzed Candidatus Desulforudis audaxviator populations. A Global location, distance and depth below surface of environmental samples from which CDA SAGs were obtained. B Average nucleotide identity in relation to geographic distance. $\mathbf{C F}_{\mathrm{ST}}$ in relation to geographic distance. The sitespecific colors of the pairwise comparisons are shown for each data point. Standard deviations and standard errors are smaller than symbols (see Table S4). 
Tau Tona Mines are within $\sim 2.7 \mathrm{~km}$ of each other and are $200 \mathrm{~km}$ from Beatrix Mine. Ground water samples from BLM1 (Fig. 1A) were collected at pressure from 755 mbls. on August 21, 2015 using a truck-mounted discrete sampler and subsampled via a sterile platinumcured silicone hose. Physical parameters (temperature, dissolved $\mathrm{O}_{2}$, conductivity, and oxidation/reduction potential were obtained using an Idronaut Sonde (GeoVista, UK). The BLM1 water samples for chemistry and other measurements were collected and analyzed as described elsewhere [20]. Samples from Byelii Yar borehole 1-R (BY-1R) were collected on April 30, 2016 (Fig. 1A). Sampling and characteristics of the water chemistry have been described previously [16, 21]. All samples for SAG analyses were preserved with 5\% glycerol and 1x TE buffer (final concentrations), frozen on site, and placed in a $-80{ }^{\circ} \mathrm{C}$ freezer the same day upon receipt at Bigelow.

\section{Single cell genomics and metagenomics}

The generation, identification, sequencing and de novo assembly of SAGs was performed at the Bigelow Laboratory for Ocean Sciences Single Cell Genomics Center-SCGC (scgc.bigelow.org). The cryopreserved samples were thawed, pre-screened through a $40 \mu \mathrm{m}$ mesh size cell strainer (Becton Dickinson) and incubated with $5 \mu \mathrm{M}$ (final concentration) SYTO-9 DNA stain (Thermo Fisher Scientific) for 10-60 min. In addition, an aliquot of the BLM1 sample was labeled with the RedoxSensor Green viability probe that detects oxidoreductase activity in sorted cells (Thermo Fisher Scientific), at a final concentration of $1 \mu \mathrm{M}$ for 20-40 min. Fluorescence-activated cell sorting, cell size estimates, cell lysis, DNA amplification with WGA-X, sequencing (using Illumina technology), de novo genome assemblies and quality control were performed as previously described [22]. This workflow was evaluated for assembly errors using three bacterial benchmark cultures with diverse genome complexity and \%GC, indicating no non-target and undefined bases and the following average frequencies of misassemblies, indels and mismatches per $100 \mathrm{Kbp}: 1.5,3.0$, and 5.0, respectively [22]. CheckM v1.0.6 was used to calculate the estimated completeness of the SAG assemblies [23]. For SAG identification, low coverage shotgun sequencing, as well as PCR-based 16S rRNA gene screens were performed as previously described [23]. All individual SAGs were deposited in MG-RAST as site-specific CDA "metagenomes" under accession numbers mg1752158 (Beatrix), mg1752155 (Mponeng), mgl752152 (Tau Tona), mg1752164 (BLM1), and mg1752161 (BY-1R).
Metagenomic samples were collected from Beatrix gold mine 4 days after the collection of samples for single cell genomics. Microbial biomass collection, total DNA and RNA extraction, sequencing and assembly were performed as previously described [19, 24, 25]. Samples from BY-1R were collected on August 05, 2014 for metagenomic studies, and total DNA extraction, sequencing and assembly were performed as previously described [16]. Contigs representing CDA were identified and mapped to the CDA MP104C MAG using BLASTN. The circularized genome was then obtained upon joining of contigs mapped on the reference genome. Illumina reads were mapped to the CDA MP104C MAG using Bowtie 2 [26]. The correctness of the assembly was verified using Bandage [27], and by mapping metagenomic reads back to the BY-1R CDA BY57 MAG.

\section{Bioinformatics}

The 16S rRNA gene sequences were aligned using SINA alignment software [28] and ClustalX [29]. Phylogenetic trees were inferred by MEGA 7.0 using the General TimeReversible Model, with Gamma distribution with invariable sites $(\mathrm{G}+\mathrm{I})$, and $95 \%$ partial deletion for 1000 replicate bootstraps. SAG assemblies were analyzed for protein-encoding regions using RAST (http://rast.nmpdr. org/) [30], and genes (protein families) were annotated with Koala (KEGG) [31] and InterProScan v5 [32]. Average nucleotide identity (ANI) was calculated using the online tools at the Kostas Lab website Environmental Microbial Genomics Laboratory (http://enve-omics.ce.gatech.edu) $[33,34]$. SAG contig alignments to the MP104C MAG were visualized in the Geneious software suite (Biomatters, Ltd.,Auckland, New Zealand). Whole genome synteny comparisons were performed with EasyFig for Mac version 2.1 [35] with tBLASTx and the filtering of small hits and annotations option. CRISPR regions were identified using the online version of CRISPR finder [36]. QUAST was used to perform genome-wide quantification of substitutions and indels [37].

Putative phage contigs were first identified using a combination of viral marker genes (viral proteins and tRNA), DNA sequence anomalies (GC skew and tetramer frequencies), and metagenomic fragment recruitment from viral and bacterial metagenomes using methods previously described by Labonté et al. [18]. Putative viral contigs were manually inspected, and contigs that did not contain obvious phage structural genes were excluded from further analysis. Viral contigs were assembled and visualized in the Geneious software suite.

Fixation indices $\left(\mathrm{F}_{\mathrm{ST}}\right)$ were calculated using the ANI among SAGs as the input metric of a formula based upon 
Hudson et al [38]:

$F_{S T}=$

$\underline{\left(1-1 / n \sum_{i=1}^{n} \text { ANI of SAGs between sites }\right)-\left(1-1 / m \sum_{j=1}^{m} \text { ANI of SAGs within sites }\right)}$ $\left(1-1 / n \sum_{i=1}^{n}\right.$ ANI of SAGs between sites)

where the within and between site ANI represent averages from a pairwise matrix of all SAGs. To define pairs of protein sequences with shared similarity between the MP104C MAG and the individual 126 SAGs, an all against all BLASTP [39] search was performed using a 95\% sequence identity cutoff [40]. The defined sequence pairs were subsequently aligned using Clustal Omega [41] with default parameters. Using the PAL2NAL tool [42], the nucleotide sequences that correspond with each of the aligned protein sequence pairs were converted into codon alignments. The resulting codon alignment pairs were estimated for synonymous and nonsynonymous substitution ratios using the YN00 program from PAML4.8 [43] with an implementation of the Yang and Nielsen 2000 method [44]. Synonymous and nonsynonymous substitution ratios were also calculated for two monocultures of Prochlorococcus marinus MIT9313 [45] and Synechococcus sp. WH8102 SAGs [46] relative to their original sequenced genomes, and current SAG co-assemblies. The finished genomes for these two strains are similar in size (2.41 and 2.64 Mbp) to that of CDA MP104C MAG (2.35 $\mathrm{Mbp}$ ). In parallel to these, a similar approach was repeated for the 7 Sulfolobus islandicus genomes [47], where pairwise estimations of synonymous and nonsynonymous substitution ratios were conducted for all genome pairs, instead of each relative to a reference. DNA polymerase modeling and fidelity testing methods are described in the Supplemental Material.

\section{Results}

\section{Global conservation of CDA genomes}

Of the 150 CDA SAGs obtained in this study, 136 yielded a 16S rRNA gene 100\% identical to that of the CDA MP104C MAG, whereas 14 had 97-99\% 16S rRNA gene identity. Of these $136 \mathrm{CDA}$ SAGs, 126 produced $>100 \mathrm{kbp}$ assemblies and were analyzed further (Table S1). The estimated completeness of the individual SAG assemblies was $<1-67 \%$, with no indications of contamination. The Mponeng 120 fracture water contained only CDA SAGs, but was collected from a different fracture, located $1.3 \mathrm{~km}$ to the southeast of the fracture that yielded CDA MP104C, and was lower in salinity than that of the MP104C fracture water [14]. The remaining four sites had relatively simple microbiomes, where CDA ranged from 10 to $40 \%$ of the SAGs (Fig. S1).

Despite the fact that the 126 CDA SAGs were obtained from North American, Eurasian and South African sites (Fig. 1A) with distinct microbial communities (Fig. S1) and physicochemical environments (e.g., temperature $37-65^{\circ} \mathrm{C}$; Eh -89 to $-337 \mathrm{mV}$; pH 6.9-8.8; and TDS 0.3-4.5 ppt; Table S2), the genomes of these SAGs were nearly identical to each other and to the CDA MP104C MAG. The ANI of all pairwise comparisons exceeded $99.5 \%$ and did not correlate with geographic distance (Fig. 1B, Table S3). An average of $94 \%$ of SAG base pairs aligned to the MP104C MAG (Table S3), with prophage regions and unique genes (e.g., phn operon) accounting for the bulk of the unaligned regions. These results are in good agreement with the limited genomic differences between CDA MP104C MAG and the isolate CDA BYF from the West Siberian borehole (ANI of 99.5\%) [17], and SAG pairwise ANI's compared to CDA BYF ( > 99.8\%). In comparison to the CDA MP104C MAG, the average nucleotide substitutions and indels of the sequenced CDA SAGs ranged from $<1$ to 448 and $<1$ to 28 per $100 \mathrm{Kbps}$, respectively (Fig. 2A, B) and did not correlate with CDA SAG completeness (Fig. S2). Although the number of substitutions and indels per $100 \mathrm{Kbps}$ in CDA SAGs were low, they were higher than the corresponding values for MIT9313 and WH8102 monocultures $(p<0.05$, ANOVA), suggesting that they were above the methodological detection limits (Fig. 2A, B). These findings stand in stark contrast to the extensive genome differences among isolates of well-characterized model organisms [48] or marine bacterioplankton in a single drop of sea water $[49,50]$. Interestingly, despite the extremely high ANI among all CDA SAGs, ANI values were higher within CDA populations than across populations, with no relationship to geographic distance (Fig. 1C, Table S4).

\section{Evidence of HGT and phage/CRISPR conservation}

We found 21 genes unique to a particular study site, often showing partial homology to other microorganisms from the subsurface (Table S5). For example, multiple CDA SAGs from Mponeng encoded an arsenite transporter, a permease, and an entire phosphonate uptake and utilization operon (phnCDEGHIJKM), which were absent in other study sites. Interestingly, phnDEIMJ had 33-70\% ANI to Betaproteobacteria Thiobacillus denitrificans, while phnCGHK had 40-50\% ANI to a Firmicutes Peptococcaceae lineage, both of which were found among SAGs from the nearby Tau Tona site (Fig. S3). Mponeng CDA SAGs also contained homologs of phnE, two of which co-localized with $\mathrm{ABC}$ phosphonate transporters. One phnE gene had $47 \%$ ANI to a Bacteriodetes lineage (Fig. S3). One CDA SAG from Beatrix and one CDA SAG from Tau Tona 


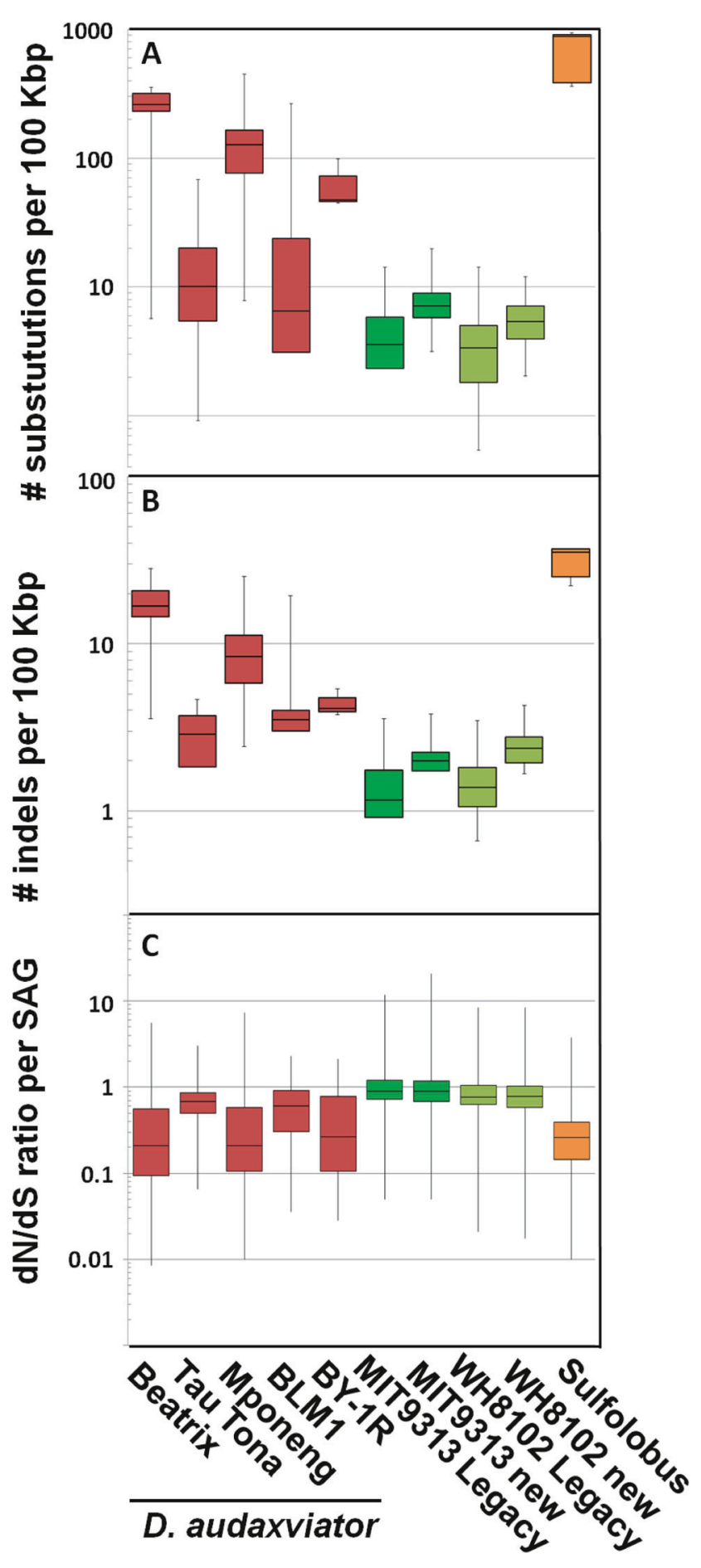

Fig. 2 Evolutionary analyses of Candidatus Desulforudis audaxviator (CDA) SAGs. Genomic variability among Candidatus Desulforudis audaxviator SAGs (red); SAGs of cyanobacteria cultures MIT9313 and WH8102 (green); and Sulfolobus islandicus genomes (orange) [47]. Variability metrics include: (A) nucleotide substitutions; (B) indels; and (C) dN/dS ratio, with respect to the MP104C reference genome. CDA SAGs are separated by study site. Cyanobacteria values, which were used as methodological controls, were calculated using two references: legacy genome sequences $[45,46]$ and new coassemblies of SAGs. contained a GDP-mannose 4,6-dehydratase ( $g m d$ ) gene with $85 \%$ ANI to a Nitrospirae genome from BLM1. These findings suggest that HGT and recombination, although infrequent, played a role in the slight genomic divergence of CDA populations from the three continents.

We found 25 regions with phage marker genes in $23 \mathrm{CDA}$ SAGs from Mponeng, BLM1, BY-R1 and Tau Tona. Of those, 10 contigs from different sites shared $100 \%$ nucleotide identity in overlapping regions with the exception of one nucleotide insert (Fig. 3A and Table S6). The presence of bacterial genes flanking at least one putative prophage region (Fig. 3A; gray arrows), the failure to recover complete phage genomes from the SAGs, and the lack of anomalies in the relationship between single cell whole genome amplification speed versus host genome recovery (Fig. S4) all suggest lysogeny rather than lytic infections, in agreement with earlier findings from the Tau Tona CDA SAGs [18].

We also identified 33 partial CRISPR regions and associated proteins, collectively containing 138 unique spacer regions across all CDA SAGs with $100 \%$ nucleotide identity. This is consistent with the comparison of the CRISPR regions of the CDA BYF genome and CDA MP104C MAG, which revealed near-complete conservation of CRISPR region order and nucleotide-level identity, with the exception of one SNP and five additional spacer regions found along the length of the array [17]. Additional spacers were not located toward the beginning of the array, where they would be inserted in response to recent viral infections [17]. CRISPR repeats and spacer regions identified in CDA MP104C MAG were also found in CDA SAGs from all three continents. Two CRISPR spacers in Tau Tona CDA SAGs had $100 \%$ sequence identity to five putative prophages in CDA SAGs from BLM1 and the CDA BYF genome (Fig. 3C). No prophage-like sequences matching this spacer were identified in Tau Tona, which is expected if the CRISPR spacer prevents infection from the corresponding phage.

\section{Discussion}

\section{Global dispersal}

CDA genomes from all three continents revealed a striking degree of conservation manifested in high ANI, few SNP's, and the conservation of prophages and CRISPRs. The latter is particularly surprising, since CRISPRs are generally considered one of the most rapidly evolving genome regions [51]. This prompted us to consider the following possible mechanisms to explain these observations: (1) cross-contamination of CDA in the lab or in the field; (2) recent dispersal between the subsurface sites via aerial transport; and (3) ancient dispersal combined with evolutionary stasis. 


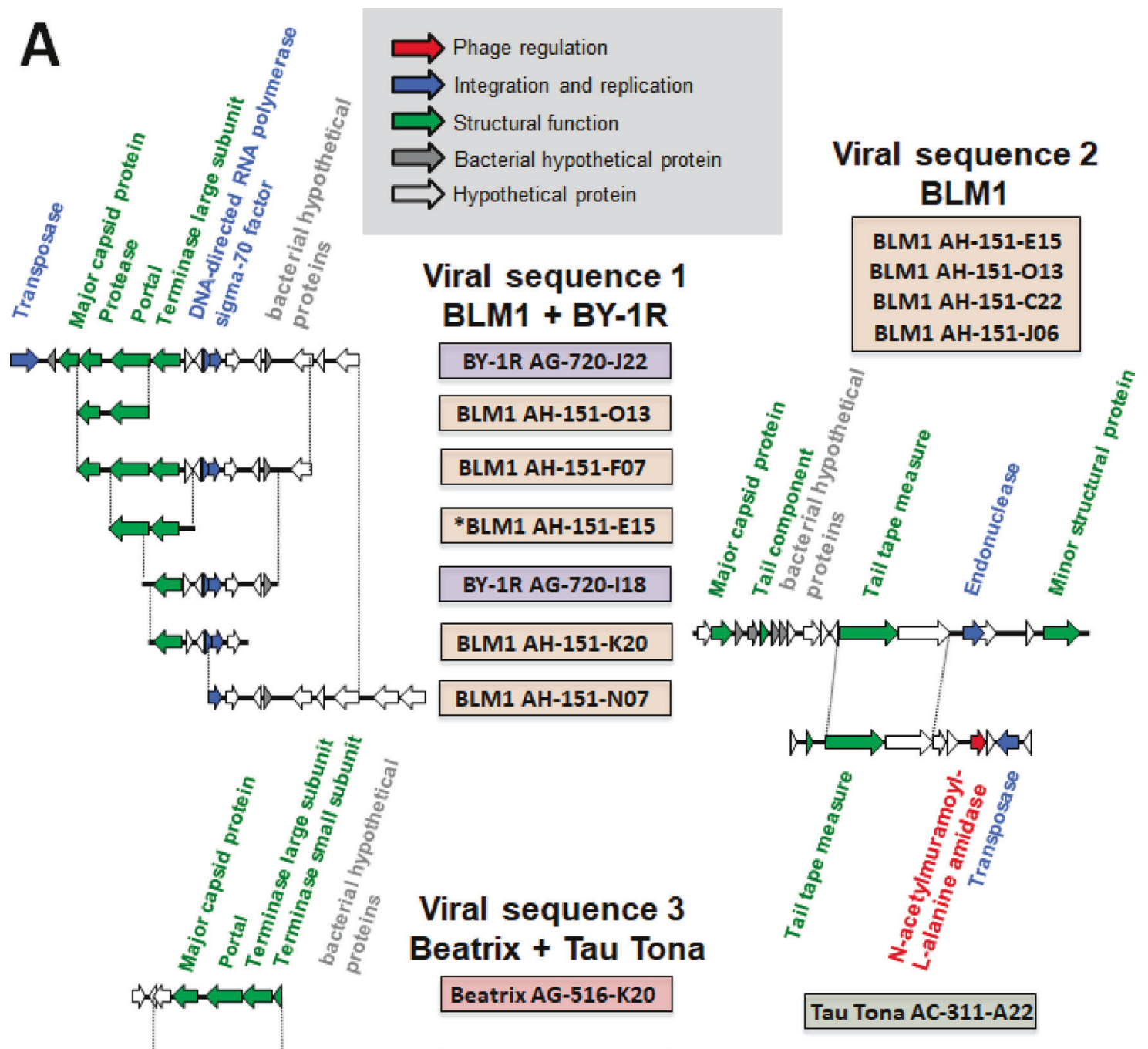

B

\section{Viral sequence 1 BLM1 + BY-1R}

AH-720-J22 BY-1R

AH-147-N07 BLM1 AH-147-K20 BLM1

AH-151-F07 BLM1

AC-311-121 Tau Tona

GATGAGCGAGATATTCAAGATCAGGATCACGAAAGACGTGATTTACGACGGCTGCGAGTATCAAGCCGGGAT GATGAGCGAGATATTCAAGATCAGGATCACGAAAGACGTGATTTACGACGGCTGCGAGTATCAAGCCGGGAT GATGAGCGAGATATTCAAGATCAGGATCACGAAAGACGTGATTTACGACGGCTGCGAGTATCAAGCCGGGAT GATGAGCGAGATATTCAAGATCAGGATCACGAAAGACGTGATTTACGACGGCTGCGAGTATCAAGCCGGGAT CRISPR spacer AAGATCAGGATCACGAAAGACGTGATTTACGACG
AH-720-J22 BY-1R AH-147-K20 BLM1 AH-151-E15 BLM1 AH-151-F07 BLM1 ACGCCGAGCGTCGGCCGGCCGTACTTTACCGTAGTGCCGTTAAACACAGTGGGCATTTCCCTGAAGATTGA ACGCCGAGCGTCGGCCGGCCGTACTTIACCGTAGTGCCGTTAAACACA GTGGGCATTTCCCTGAAGATTGA ACGCCGAGCGTCGGCCGGCCGTACTTTACCGTAGTGCCGTTAAACACAGTGGGCATTTCCCTGAAGATTGA ACGCCGAGCGTCGGCCGGCCGTACTTIACCGTAGTGCCGTTAAACACA GTGGGCATTTCCCTGAAGATTGA AC-311-I21 Tau Tona CRISPR spacer ACCGTAGTGCCGTTAAACACAGTGGGCATTTCCCT

Fig. 3 Examples of identical prophage and CRISPR sequences in SAGs from geographically distant locations (see Table S6 for a complete list). A Alignments of SAG prophages sourced from multiple field sites. Viral sequence 1 was found in SAGs from BLM1 (North America) and BY-1R (Eurasia). Viral sequence 2 was found in SAGs from BLM1 (North America) and Tau Tona (Africa). Viral sequence 3 was found in SAGs from Beatrix (Africa) and Tau Tona.
Dotted lines show regions with $100 \%$ nucleotide identity. Asterisk in BLM1 AH-151-E15 represents the one single nucleotide insert in the alignments. SAGs are color-coded by site; BY-1R, purple; BLM1, orange; Tau Tona, green; and Beatrix, red. B Prophage contigs from BLM1 (North America) and BY-1R (Eurasia) aligned to two different CRISPR spacer regions from Tau Tona (Africa). 
SAG analyses were performed in a cleanroom environment that has been consistently proven to prevent sample cross-contamination [22, 52], and CDA SAG sample analyses were separated by several years. For example, the CDA SAG samples from Tau Tona that contain viral contigs and CRISPR sequences identical to viral elements in CDA SAG samples from BLM1 and BY-R1 were sorted, amplified and sequenced in 2012, whereas those from BLM1 and BY-R1 were analyzed in 2016 and 2017, respectively. The sample containing the CDA MP104C MAG, which has CRISPR elements identical to those of the BLM1 SAGs and CDA BYF, was collected from a freshly drilled borehole in 2002, DNA was extracted in 2003 at the Princeton University lab and the metagenome sequenced at the Joint Genome Institute (JGI) in 2005. The CDA SAG samples did not arrive at the Princeton University lab until 2012-2014 and were shipped directly to SCGC upon arrival without opening. Even if one speculates that the CDA SAGs were cross-contaminated, there is no way to explain the $100 \%$ identity in CRISPR elements between the CDA MP104C MAG sequenced at JGI in the U.S. in 2005 and the CDA BYF culture isolated and sequenced in Russia in 2018 as cross-contamination.

Next, we examined the possibility of anthropogenic crosscontamination in the field. To the best of our knowledge, there has never been a cross-use of drilling or sampling equipment among our South African mine drilling contractors, the U.S. Department of Energy well drilling contractors for BLM1 completed in 2007, and Russian oil well drilling contractors for the Byelii Yar oil well, which was drilled in 1962. In South Africa, CDA has been detected in many deep ground water and fracture water samples across the Witwatersrand Basin [53-55] in boreholes drilled between the mid-1990's and 2011, but it has never been found in water used for mining operations [54]. Likewise, investigators involved in the collection of the Siberian sample never visited our sites in South Africa and California, and vice versa. Only one investigator who collected samples from South African sites also did so from the Californian site, but after a 17-year interlude. We conclude that sampling cross-contamination cannot explain the high degree of genome similarity among the analyzed CDA populations on three continents.

We assessed the possibility of recent natural dispersal as an explanation for CDA genome conservation. For instance, Sulfolobus islandicus, Sulfolobus acidocaldaria and Thermus species from globally distributed hot springs revealed similar genomes, but contained from $10 \times$ to $100 \times$ higher substitution frequencies and indels [47, 56-58] than CDA genomes. Sulfolobus acidocaldaria and Thermus CRISPR spacers were found to be mostly conserved across multiple continents, though the number of shared spacers decreased with increasing geographic distance [58], unlike those of the CDA genomes. If the Sulfolobus islandicus genome SNP's were acquired over an estimated 910,000 year period since population separation by aerial dispersal, which is based upon the ages of the volcanic formations, then the average rate of nucleotide substitution is a minimum of $4.7 \times 10^{-9}$ substitutions per site per year [47]. Using this rate, Karnachuk et al. [17] estimated a divergence time of $\sim 2800$ to 3.1 million years for the MP104C MAG and CDA BYF genome. To fit this time frame, Karnachuk et al. [17] hypothesized that CDA were dispersed aerially as spores. Although CDA BYF does exhibit some short term tolerance of microaerophilic conditions [17], CDA lacks $\mathrm{O}_{2}$ protection genes [14], suggesting that frequent, long-distance migration over surface environments (e.g., via air and water) in vegetative form is unlikely.

For additional evidence of CDA global dispersal by air, we searched for 16S rRNA gene sequences with $\geq 99 \%$ identity to the MP104C MAG in public databases: NCBI Genbank, RDP II [59], SILVA [28], Greengenes [60], the JGI Integrated Microbial Genomes database [61], Integrated Microbial Next Generation Sequencing (IMNGS) [62], iMicrobe [63], the Earth Microbiome Project [64] and Tara Ocean Project [65]. Most commonly used PCR primers for bacterial 16S rRNA genes have high estimated binding efficiency for CDA, and only a less commonly used primer 341-f had mismatches (Table S7). Excluding the sites reported in this study, we found only 1854 partial 16 S rRNA sequences with $\geq 99 \%$ nucleotide identity to CDA in all IMNGS metagenomes and other datasets. With the exception of several reads present in seafloor sediments near a methane seep off the coast of Oregon [66] and one read in Guaymas Basin sediment [67], all reads were found in samples from continental or island subsurface sites 400-1200 m deep (Table S8). Most of the reads originated from a Pleistocene basaltic aquifer in Iceland [68, 69]; an additional location in the same Paleozoic dolomite aquifer accessed by BLM1 and in the overlying Miocene volcanic units in Nevada [20]; in Neogene-Paleogene and Cretaceous gas reservoirs in Japan [70, 71]; and in Upper Cretaceous coal bed gas reservoirs in Alberta, Canada [72, 73]. A single CDA sequence read originated from a biofilm in an anaerobic geothermal reactor in Denmark; which could have originated from subsurface geothermal water that supplied the facility [74]. CDA was not detected in any hot springs, including those in South Africa [75]. Even though a few CDA-like $16 \mathrm{~S}$ rRNA sequences were detected at one marine methane seep [66], deposition at our mid-continent sites would still require aerial transport, which should leave a footprint in soil sites, but no CDA have been reported in soils. Given the large scale of current 16S rRNA gene surveys, in particular the Earth Microbiome project that encompasses 2.2 billion 16S rRNA gene sequences from 27,751 environmentally diverse and globally distributed samples, and the tens of thousands of environmental datasets in IMNGS, our findings suggest no current dispersal of CDA by air. The one caveat to this 
evidence is that aerial dispersal could have occurred tens to hundreds of thousands of years ago and would be undetectable today given the survival time of DNA in soil [76]. Two other arguments oppose the aerial dispersal as spores; (1) the 2800-3.1-million-year separation age is younger than the 20 myr old ground water ages for some of the CDA-bearing South African sites (Fig. S5); and (2) thermophilic spore lifetimes are on the order of hundreds of years [77, 78], which is far shorter than the ground water ages for the CDA-bearing South African sites, implying that CDA spores would be dead before reaching subsurface fractures to germinate.

The final possibility is that CDA has been dispersed in the subsurface via migration along ground water flow paths in a primarily vegetative state. This is unlikely, given CDA cannot grow at sea water salinity [17] and has not been reported in deep sea vents [79]. CDA is distributed over a distance of at least $300 \mathrm{~km}$ in the South African Witwatersrand Basin by fluid flow, primarily along fractures, and likely has been residing there for at least 20 myr (Fig. S5). However, CDA, like all subsurface microorganisms, likely spend most of their time attached to mineral surfaces, perhaps in microcolonies, and the dispersal times across even thousands of kilometers may require tens to hundreds of millions of years.

$\mathrm{F}_{\mathrm{ST}}$ is a metric commonly used in ecology to measure genetic exchange between eukaryotic populations. Although the CDA populations are genetically very similar (Table S4 and Fig. S6), $\mathrm{F}_{\mathrm{ST}}$ values demonstrated they are genetically divergent from one another (Fig. 1C). We are not aware of other $\mathrm{F}_{\mathrm{ST}}$ analyses of natural microbial populations, but $\mathrm{F}_{\mathrm{ST}}$ values of $>0.9$ suggest a very limited exchange between CDA populations after separation [38].

A minimum time for separation of the CDA habitats from our study sites is set by the $\sim 165 \mathrm{Ma}$ separation time for Africa from Laurentia (North America + Eurasia), with the formation of the Central Atlantic rift zone [80], and the $\sim 55$ Ma separation time for North America from Eurasia with the formation of the North Atlantic rift zone [81]. At $\sim 47 \mathrm{Ma}$, the Jan Mayen Microcontinent broke away from Greenland and was buried beneath the Icelandic Plateau [81]. Japan was connected to the Asian continent at the time the CDA-bearing sediments were deposited, and did not split from Asia until $\sim 15 \mathrm{Ma}$ [82]. Although the separation of our CDA populations could have occurred during the breakup of supercontinents prior to Gondwana, even this most recent separation time requires remarkable genome stability.

\section{Potential drivers of genome stability}

In order to evaluate the potential mechanisms leading to the unusual genome stability of CDA, we considered dormancy as a spore [14, 17], purifying selection and high-fidelity DNA replication and repair mechanisms. Scanning electron microscopy demonstrated the predominance of vegetative cells in the MP104C MAG sample, which contained only CDA [14], while metatranscriptome analyses of the Beatrix fracture water [19] indicated that CDA were actively expressing sulfate reducing genes (Table S9). The in situ turnover time of CDA biomass at the MP104C MAG site was estimated from aspartic acid racemization at $<1$ year [13]. Analyses of the CDA BYF isolate revealed it rarely formed spores [17]. In this study, we found that CDA from BLM1 expressed oxidoreductase activity and readily stained with a nucleic acid dye (Fig. 4), providing further support for their in situ vegetative state. We were unable to analyze oxidoreductase activity for the South African and BYR-1 samples, as the protocol was not in place when the samples were processed. Recent data show that spore lifetimes of thermophiles are not significantly longer than those of vegetative cells under starvation conditions at optimal growth temperatures [77]. In addition, due to the amino acid racemization-induced mortality [77], dormancy extending beyond a few years at thermophilic temperatures is unlikely [13]. The collective evidence from the various techniques suggests that the studied CDA populations contained metabolically active and replicating cells, which, depending upon the temperature, suggests doubling times of no more than $<1-10$ years.

Next, we considered the possibility of selective forces playing a role in the conservation of CDA genomes. The genome-wide ratio of non-synonymous to synonymous substitutions (dN/dS) averaged between 0.21 and 0.68 in the studied populations (Fig. 2C). These values are higher than in most other microbial populations hypothesized to be under purifying selection, such as marine Marinimicrobia (<0.1) [83], Cyanobacteria (0.03-0.08) [84] and Alphaproteobacteria (0.00-0.25) [85], and diverse Gammaproteobacteria (average of 0.05) [86]. We conclude that the relatively high $\mathrm{dN} / \mathrm{dS}$ of the analyzed CDA populations provides no evidence for unusually strong purifying selection in this lineage.

We examined DNA repair mechanisms and polymerase fidelity as factors potentially contributing to genome stability (Table S10). CDA SAGs and the MP104C MAG encode at least seven DNA repair mechanisms: MutL-MutS system, UvrABC system, DinG, RecA, RadA, RecFOR, RecBCD, UvrD; as well as other nucleases involved in end-joining, internal excision, and end trimming. Furthermore, CDA encode DNA-binding proteins (e.g., HU-beta), which may also reduce mutation rates $[87,88]$. CDA genomes encode DNA polymerases I ( pol), III, IV, and X. The polymerase I of CDA contains multiple non-overlapping domains (starting from N-terminus): 5'-3' exonuclease domain, Ribonuclease $\mathrm{H}$ superfamily domain, and palm domain of family A DNA polymerase (Fig. 5A, B). The Ribonuclease $\mathrm{H}$ superfamily domain is responsible for the $3^{\prime}-5^{\prime}$ proofreading exonuclease activity in E. coli, whereas it is inactive in Thermus aquaticus [89]. Structural analysis, however, indicated that the CDA 
A. RedoxSensor Green fluorescence (viability)

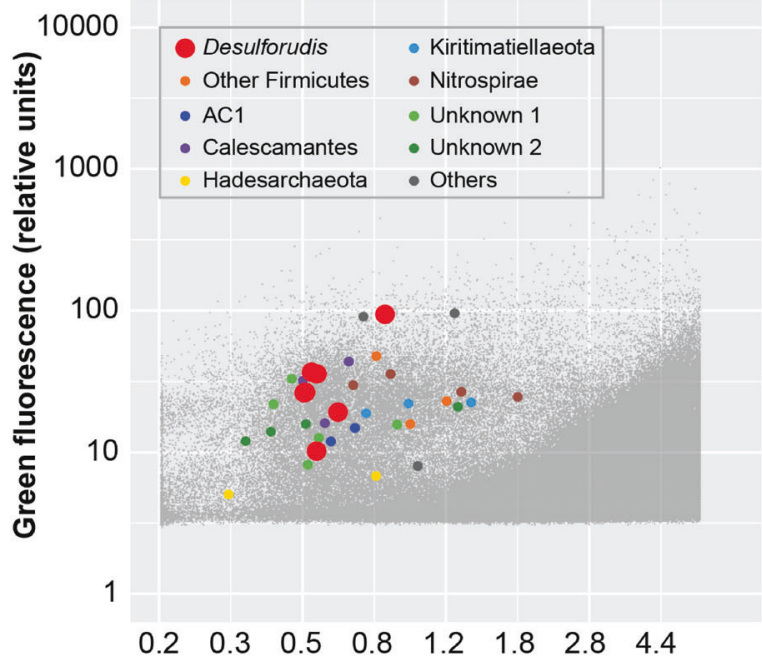

Estimated cell diameter $(\mu \mathrm{m})$

\section{B. Syto-9 fluorescence (nucleic acid content)}

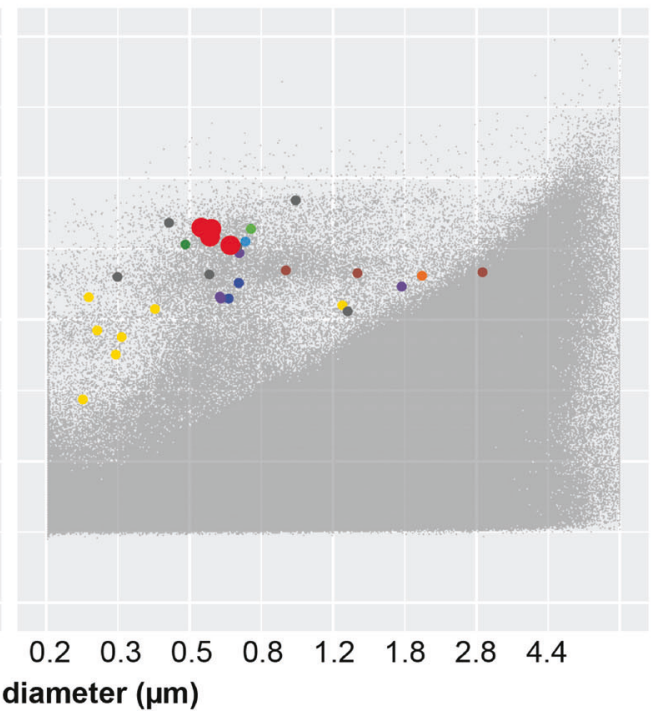

Fig. 4 Flow-cytometric characterization of microorganisms from BLM1. A Oxidoreductase activity and (B) nucleic acid content analyzed by flow cytometry at BLM1 site in California. Identified cells are color-coded, with CDA colored red and enlarged for visual recognition. The $Y$-axis is fluorescence. The $X$-axis is estimated diameter of the sorted cells, derived from the forward light scatter.

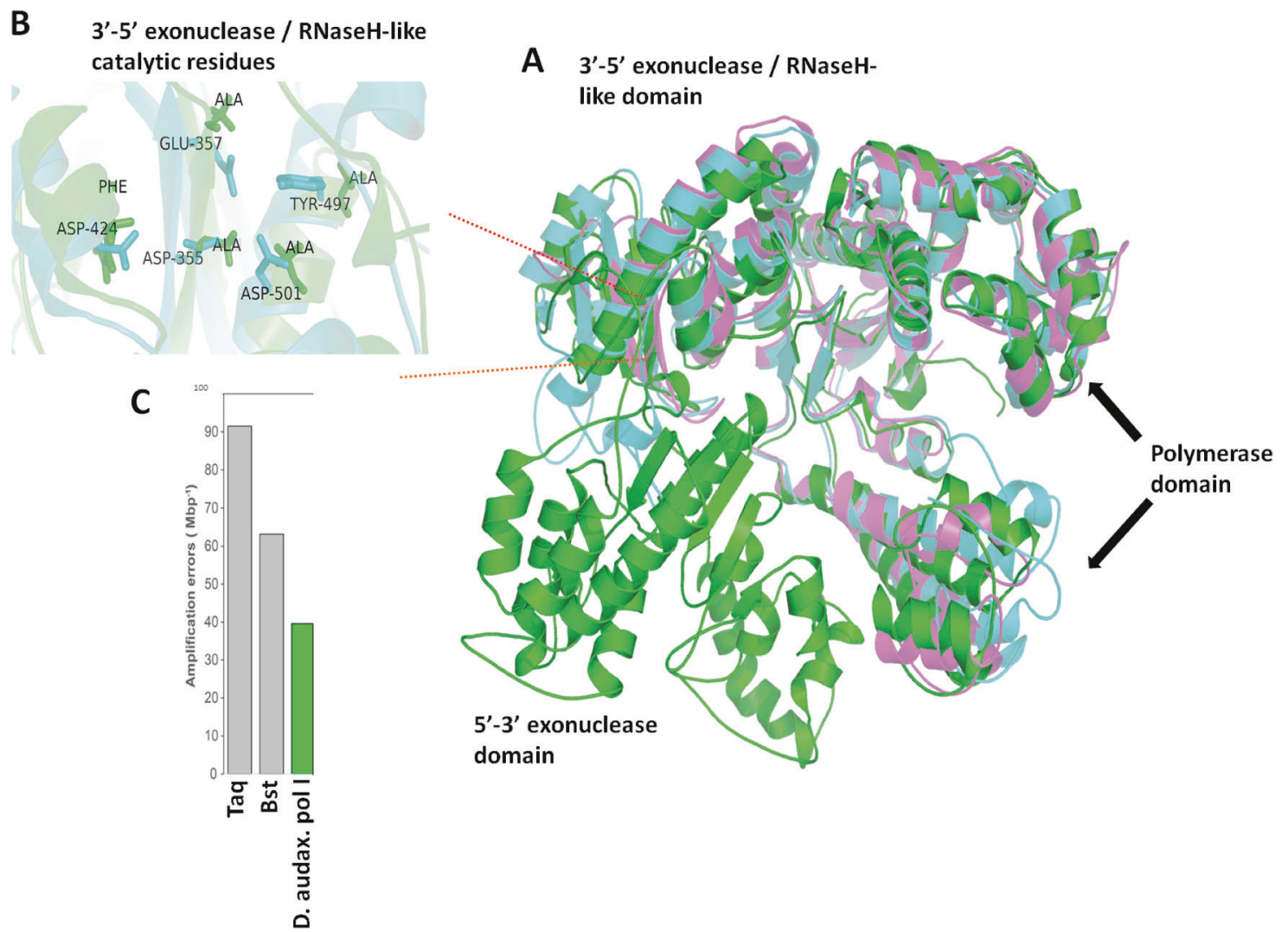

Fig. 5 Candidatus Desulforudis audaxviator DNA polymerase I model. A Models of the large fragment of polymerase I of CDA (green), large Klenow fragment of E. coli DNA polymerase I (cyan) and Thermus aquaticus DNA polymerase I (purple). See
Tables S10A-C for corresponding protein and domain IDs. B Enlarged view of the $3^{\prime}-5^{\prime}$ exonuclease site and RNaseH-like catalytic residues. C Experimental evaluation of the fidelity of CDA DNA polymerase I, in comparison to Taq and Bst polymerases. 
polymerase I is unlikely to possess $3^{\prime}-5^{\prime}$ proofreading activity (Figs. S7 and S8).

In order to experimentally evaluate the fidelity of CDA DNA polymerases I, IV and X, we synthesized them by heterologous expression in E. coli. Only polymerase I was obtained in a water-soluble form and could be analyzed further. This polymerase phylogenetically clusters with a mixed group of Thermoanaerobacterales and deeply branching Clostridiales, with the closest ortholog being from Desulfovirgula thermocuniculi (Thermoanaerobacterales) (Fig. S9). Under the applied in vitro conditions, this polymerase produced 40 mismatches per one Mbp, which demonstrates higher fidelity than Taq and Bst (Fig. 5C), but lower than some of the enzymes utilized in isothermal DNA amplification, such as phi29 [90]. However, the hypothesized high fidelity of CDA DNA replication could still be a result of factors other than polymerase I, such as highly accurate DNA polymerases IV and $\mathrm{X}$ or sophisticated DNA repair mechanisms.

\section{Concluding remarks}

The collective evidence suggests that minimal evolution has taken place in the studied CDA populations from African, Eurasian and North American sites since their separation from the ancestral population. Based upon our analyses, the most likely scenario is that this separation occurred between 165 and $55 \mathrm{Ma}$, during the breakup of Pangea. High fidelity of DNA replication and repair mechanisms remains the most plausible mechanisms behind this extreme genome conservation, although we were unable to confirm this in vitro.

CDA presents a stark contrast to the current model organisms in microbial evolutionary studies, which are found to develop adaptive traits over far shorter periods of time [2]. Our findings suggest that the separated CDA populations are more analogous to Darwin's finches with subtle variations in color as opposed to large differences in beak size, and call for a re-evaluation of some of the explicit and implicit assumptions about microbial evolution. For example, long, unknown periods of evolutionary stasis may impact the scaling of the molecular clock [1], the topology of inferred phylogenetic relationships, and the development of standardized divergence thresholds for a streamlined microbial taxonomy $[91,92]$. The hypothesized high fidelity of CDA DNA replication and repair mechanisms may find practical applications in biotechnology. Since the subsurface harbors an estimated $10 \%$ of planet's microbial biomass and a large fraction of its biodiversity, it may be expected that CDA is not the only living microbial fossil in this vast environment, potentially offering unique sources of information about the history of life.
Acknowledgements We thank the staff of the Bigelow Laboratory Single Cell Genomics Center for the generation of single cell genomics data. We also thank Sibanye Gold Limited and AngloGold Ashanti Limited and the mine managers and employees of Beatrix, Mponeng and Tau Tona gold mines for their assistance in sampling. Inyo-BLM 1 samples were obtained under scientific research permit DEVA-2013SCI-0069 from the U.S. National Park Service (NPS). We thank Richard Friese, Josh Hoines, and Kevin Wilson of the NPS along with Alisa Lembke and the Inyo County, CA Planning Commission for site access. Thanks also to: John Healey, Brad Lyles, Scott HamiltonBrehm, Chuck Russell, and Sean Mullin for logistical contributions; to John Bredehoeft and Michael King of The Hydronamics Group for hydrogeological context; and to Bill Wilborn and the USDOE UGTA program for use of their borehole logging system. We thank Raphael Laurenceau, Paul Berube and Sallie Chisholm (Massachusetts Institute of Technology) for providing monoclonal cultures of Prochlorococcus marinus MIT9313 and Synechococcus sp. WH8102. We also thank Mohammed El-Kebir (University of Illinois Urbana-Champaign) and Thane Papke (University of Connecticut) for advice on population genetics analyses and Birute Gagiliene for overseeing the polymerase laboratory analyses. This work was supported by NSF awards DEB1441717 (to RS, TC and DM) and OIA-1826734 (to RS and DM), NASA Astrobiology Institute award NNA13AA92A (to DM), Russian Science Foundation grant 14-14-01016 and 19-14-00245 (to NR), and Nevada NASA Space Grant Consortium Graduate Fellowship NNX15AI02H (to JDS).

\section{Compliance with ethical standards}

Conflict of interest The authors declare no competing interests.

Publisher's note Springer Nature remains neutral with regard to jurisdictional claims in published maps and institutional affiliations.

Open Access This article is licensed under a Creative Commons Attribution 4.0 International License, which permits use, sharing, adaptation, distribution and reproduction in any medium or format, as long as you give appropriate credit to the original author(s) and the source, provide a link to the Creative Commons license, and indicate if changes were made. The images or other third party material in this article are included in the article's Creative Commons license, unless indicated otherwise in a credit line to the material. If material is not included in the article's Creative Commons license and your intended use is not permitted by statutory regulation or exceeds the permitted use, you will need to obtain permission directly from the copyright holder. To view a copy of this license, visit http://creativecommons. org/licenses/by/4.0/.

\section{References}

1. Ochman H, Wilson AC. Evolution in bacteria: evidence for a universal substitution rate in cellular genomes. J Mol Evolution. 1987;26:74-86.

2. Cooper VS, Lenski RE. The population genetics of ecological specialization in evolving Escherichia coli populations. Nature 2000;407:736-9.

3. Gibson B, Eyre-Walker A. Investigating evolutionary rate variation in bacteria. J Mol Evol. 2019;87:317-26.

4. Parks DH, Rinke C, Chuvochina M, Chaumeil P-A, Woodcroft BJ, Evans PN, et al. Recovery of nearly 8,000 metagenomeassembled genomes substantially expands the tree of life. Nat Microbiol. 2017;2:1533-42. 
5. Hug LA, Baker BJ, Anantharaman K, Brown CT, Probst AJ, Castelle CJ, et al. A new view of the tree of life. Nat Microbiol. 2016;1:16048.

6. Rinke C, Schwientek P, Sczyrba A, Ivanova NN, Anderson IJ, Cheng J-F, et al. Insights into the phylogeny and coding potential of microbial dark matter. Nature. 2013;499:431-7.

7. Kallmeyer J, Pockalny R, Adhikari RR, et al. S. Global distribution of microbial abundance and biomass in subseafloor sediment. Proc Natl Acad Sci USA. 2012;109:16213-6.

8. Magnabosco C, Lin L-H, Dong H, Bomberg M, Ghiorse W, StanLotter $\mathrm{H}$, et al. The biomass and biodiversity of the continental subsurface. Nat Geosci. 2018;11:707-17. https://doi.org/10.1038/ s41561-018-0221-6.

9. Castelle CJ, Wrighton KC, Thomas BC, Hug LA, Brown CT, Wilkins MJ, et al. Genomic expansion of domain archaea highlights roles for organisms from new phyla in anaerobic carbon cycling. Curr Biol. 2015;25:690-701.

10. Brown CT, Hug LA, Thomas BC, Sharon I, Castelle CJ, Singh A, et al. Unusual biology across a group comprising more than $15 \%$ of domain Bacteria. Nature 2015;523:208-11.

11. Beam JP, Becraft ED, Brown JM, Schulz F, Jarett JK, Bezuidt O, et al. Ancestral Absence of Electron Transport Chains in Patescibacteria and DPANN. Front Microbiol. 2020;11:1848 https:// doi.org/10.3389/fmicb.2020.01848. PubMed PMID: 33013724; PubMed Central PMCID: PMCPMC7507113

12. Braun S, Mhatre SS, Jaussi M, Røy H, Kjeldsen KU, Pearce C, et al. Microbial turnover times in the deep seabed studied by amino acid racemization modelling. Sci Rep. 2017;7:5680.

13. Onstott TC, Magnabosco C, Aubrey AD, Burton AS, Dworkin JP, Elsila JE, et al. Does aspartic acid racemization constrain the depth limit of the subsurface biosphere? Geobiology. 2014;12: $1-19$.

14. Chivian D, Alm E, Brodie E, Culley D, Dehal P, DeSantis T, et al. Environmental genomics reveals a single species ecosystem deep within the Earth. Science. 2008;322:275-8. doi: 0.1126/ science. 1155495

15. Kadnikov VV, Frank YA, Mardanov AV, Beletsky AV, Karnachuk OV, Ravin NV. Metagenome of the Siberian Underground Water Reservoir. Genome Announc. 2017;5:e01317-

16. Kadnikov VV, Mardanov AV, Beletsky AV, Banks D, Pimenov $\mathrm{NV}$, Frank YA, et al. A metagenomic window into the $2 \mathrm{~km}$-deep terrestrial subsurface aquifer revealed multiple pathways of organic matter decomposition. FEMS Microbiol Ecol. 2018;94: fiy 152.

17. Karnachuk OV, Frank YA, Lukina AP, Kadnikov VV, Beletsky AV, Mardanov AV, et al. Domestication of previously uncultivated Candidatus Desulforudis audaxviator from a deep aquifer in Siberia sheds light on its physiology and evolution. ISME J. 2019;13:1947-59.

18. Labonté J, Field E, Lau M, Chivian D, vanHeerden E, Wommack $\mathrm{KE}$, et al. Single cell genomics indicates horizontal gene transfer and viral infections in a deep subsurface Firmicutes population. Front Microbiol. 2015;6:349.

19. Lau MCY, Kieft TL, Kuloyo O, Linage B, Heerden EV, Lindsay MR, et al. Deep-subsurface community dependent on syntrophy is dominated by sulfur-driven autotrophic denitrifiers. Proc Natl Acad Sci USA. 2016;113:E7927-E36. https://doi.org/10.1073/pna s.1612244113.

20. Mullin SW, Wanger G, Kruger BR, Sackett JD, Hamilton-Brehm $\mathrm{SD}$, Bhartia R, et al. Patterns of in situ mineral colonization by microorganisms in a $\sim 60^{\circ} \mathrm{C}$ deep continental subsurface aquifer. Frontiers in Microbiology. 2020;11:2573.

21. Banks D, Frank YA, Kadnikov VV, Karnachuk OV, Watts M, Boyce A, et al. Hydrochemical data report from sampling of two deep abandoned hydrocarbon exploration wells: Byelii Yar and Parabel, Tomsk Oblast, Western Siberia, Russian Federation.
NGU Report No. 2014.034. (Geological Survey of Norway, Trondheim, Norway, 2014).

22. Stepanauskas R, Fergusson EA, Brown J, Poulton NJ, Tupper B, Labonté JM, et al. Improved genome recovery and integrated cellsize analyses of individual uncultured microbial cells and viral particles. Nat Commun. 2017;8:84.

23. Parks DH, Imelfort M, Skennerton CT, Hugenholtz P, Tyson GW. CheckM: assessing the quality of microbial genomes recovered from isolates, single cells, and metagenomes. Genome Res. 2015;25:1043-55

24. Lau CYM, Cameron C, Magnabosco C, Brown CT, Schilkey F, Grim $\mathrm{S}$, et al. Phylogeny and phylogeography of functional genes shared among seven terrestrial subsurface metagenomes reveal Ncycling and microbial evolutionary relationships. Front Microbiol. 2014;5:531.

25. Magnabosco C, Ryan K, Lau MCY, Kuloyo O, Lollar BS, Kieft $\mathrm{TL}$, et al. A metagenomic window into carbon metabolism at $3 \mathrm{~km}$ depth in Precambrian continental crust. ISME J. 2016;10:730-41. https://doi.org/10.1038/ismej.2015.150.

26. Langmead B, Salzberg SL. Fast gapped-read alignment with Bowtie 2. Nat Methods. 2012;9:357-9.

27. Wick RR, Schultz MB, Zobel J, Holt KE. Bandage: interactive visualization of de novo genome assemblies. Bioinformatics 2015;31:3350-2.

28. Pruesse E, Peplies J, Glockner FO. SINA: accurate highthroughput multiple sequence alignment of ribosomal RNA genes. Bioinformatics. 2012;28:1823-9.

29. Larkin MA, Blackshields G, Brown NP, Chenna R, McGettigan $\mathrm{PA}, \mathrm{McW}$ illiam $\mathrm{H}$, et al. Clustal $\mathrm{W}$ and Clustal $\mathrm{X}$ version 2.0. Bioinformatics. 2007;23:2947-8.

30. Aziz RK, Bartels D, Best AA, DeJongh M, Disz T, Edwards RA, et al. The RAST Server: rapid annotations using subsystems technology. BMC Genom. 2008;9:75.

31. Kanehisa M, Sato Y, Morishima K. BlastKOALA and GhostKOALA: KEGG tools for functional characterization of genome and metagenome sequences. J Mol Biol. 2016;428:726-31.

32. Jones P, Binns D, Chang H-Y, Fraser M, Li W, McAnulla C, et al. InterProScan 5: genome-scale protein function classification. Bioinformatics 2014;30:1236-40.

33. Goris J, Konstantinidis KT, Klappenbach JA, Coenye T, Vandamme P, Tiedje JM. DNA-DNA hybridization values and their relationship to whole-genome sequence similarities. Int J Syst Evolut Microbiol. 2007;57:81-91.

34. Rodriguez-R LM, Konstantinidis KT. Bypassing cultivation to identify bacterial species. Microbe. 2014;9:111-8.

35. Sullivan MJ, Petty NK, Beatson SA. Easyfig: a genome comparison visualizer. Bioinformatics. 2011;27:1009-10.

36. Grissa I, Bouchon P, Pourcel C, Vergnaud G. On-line resources for bacterial micro-evolution studies using MLVA or CRISPR typing. Biochimie 2008;90:660-8.

37. Gurevich A, Saveliev V, Vyahhi N, Tesler G. QUAST: quality assessment tool for genome assemblies. Bioinformatics 2013;29: 1072-5.

38. Hudson RR, Slatkint M, Maddison WP. Estimation of levels of gene flow from DNA sequence data. Genetics. 1992;132:583-9.

39. Altschul SF, Gish W, Miller W, Myers EW, Lipman DJ. Basic local alignment search tool. J Mol Biol. 1990;215:403-10.

40. Page AJ, Cummins CA, Hunt M, Wong VK, Reuter S, Holden MTG, et al. Roary: rapid large-scale prokaryote pan genome analysis. Bioinformatics 2015;31:3691-3.

41. Sievers F, Wilm A, Dineen D, Gibson TJ, Karplus K, Li W, et al. Fast, scalable generation of high-quality protein multiple sequence alignments using Clustal Omega. Mol Syst Biol. 2011;7:539.

42. Suyama M, Torrents D, Bork P. PAL2NAL: robust conversion of protein sequence alignments into the corresponding codon alignments. Nucleic Acids Res. 2006;34:W609-W12. 
43. Yang Z. PAML 4: phylogenetic analysis by maximum likelihood. Mol Biol Evol. 2007;24:1586-91.

44. Yang Z, Nielsen R. Estimating synonymous and nonsynonymous substitution rates under realistic evolutionary models. Mol Biol Evol. 2000;17:32-43.

45. Rocap G, Larimer FW, Lamerdin J, Malfatti S, Chain P, Ahlgren NA, et al. Genome divergence in two Prochlorococcus ecotypes reflects oceanic niche differentiation. Nature. 2003;424:1042-7.

46. Palenik B, Brahamsha B, Larimer FW, Land M, Hauser L, Chain $\mathrm{P}$, et al. The genome of a motile marine Synechococcus. Nature 2003;424:1037-42.

47. Reno ML, Held NL, Fields CJ, Burke PV, Whitaker RJ. Biogeography of the Sulfolobus islandicus pan-genome. Proc Natl Acad Sci USA 2009;106:8605-10.

48. Welch RA, Burland V, Plunkett G, III, Redford P, Roesch P, Rasko D. et al. Extensive mosaic structure revealed by the complete genome sequence of uropathogenic Escherichia coli. Proc Natl Acad Sci Usa. 2002;99:17020-4.

49. Swan BK, Tupper B, Sczyrba A, Lauro FM, Martinez-Garcia M, González JM, et al. Prevalent genome streamlining and latitudinal divergence of planktonic bacteria in the surface ocean. Proc Natl Acad Sci USA. 2013;110:11463-8.

50. Kashtan N, Roggensack SE, Berta-Thompson JW, Grinberg M, Stepanauskas R, Chisholm SW. Fundamental differences in diversity and genomic population structure between Atlantic and Pacific Prochlorococcus. ISME J. 2017;11:1997-2011.

51. Tyson GW, Banfield JF. Rapidly evolving CRISPRs implicated in acquired resistance of microorganisms to viruses. Environ Microbiol. 2008;10:200-7.

52. Pachiadaki MG, Brown JM, Brown J, Bezuidt O, Berube PM, Biller SJ, et al. Charting the complexity of the marine microbiome through single-cell genomics. Cell. 2019;179:1623-35.e11.

53. Lin LH, Wang P-L, Rumble D, Lippmann-Pipke J, Boice E, Pratt LM, et al. Long-term sustainability of a high-energy, low-diversity crustal biome. Science. 2006;314:479-82.

54. Gihring TM, Moser DP, Lin L-H, Davidson M, Onstott TC, Morgan L, et al. The distribution of microbial taxa in the subsurface water of the Kalahari Shield, South Africa. Geomicrobiol J. 2006;23:415-30.

55. Moser DP, Gihring TM, Brockman FJ, Fredrickson JK, Balkwill DL, Dollhopf ME, et al. Desulfotomaculum spp. and Methanobacterium spp. Dominate 4-5km Deep Fault. Appl Environ Microbiol. 2005;71:8773-83.

56. Mao D, Grogan D. Genomic evidence of rapid, global-scale gene flow in a Sulfolobus species. ISME J. 2012;6:1613-6.

57. Anderson RE, Kouris A, Seward CH, Campbell KM, Whitaker RJ. Structured populations of Sulfolobus acidocaldarius with susceptibility to mobile genetic elements. Genome Biol Evol. 2017;9:1699-710.

58. Lopatina A, Medvedeva S, Artamonova D, Kolesnik M, Sitnik V, Ispolatov Y, et al. Natural diversity of CRISPR spacers of Thermus: evidence of local spacer acquisition and global spacer exchange. Philos Trans R Soc Lond B Biol Sci. 2019;374:20180092.

59. Wang Q, Garrity GM, Tiedje JM, Cole JR. Naive Bayesian classifier for rapid assignment of rRNA sequences into the new bacterial taxonomy. Appl Environ Microbiol. 2007;73:5261-7.

60. McDonald D, Price MN, Goodrich J, Nawrocki EP, DeSantis TZ, Probst A, et al. An improved Greengenes taxonomy with explicit ranks for ecological and evolutionary analyses of bacteria and archaea. ISME J. 2012;6:610-8.

61. Markowitz VM, Chen I-MA, Palaniappan K, Chu K, Szeto E, Grechkin Y, et al. IMG: the Integrated Microbial Genomes database and comparative analysis system. Nucleic Acids Res. 2012;40:D115-D22.

62. Lagkouvardos I, Joseph D, Kapfhammer M, Giritli S, Horn M, Haller D, et al. IMNGS: a comprehensive open resource of processed 16S rRNA microbial profiles for ecology and diversity studies. Sci Rep. 2016;6:33721.

63. Hurwitz B. iMicrobe: advancing clinical and environmental microbial research using the iPlant cyberinfrastructure. San Diego, CA: Plant and Animal Genome XXII Conference; 2014.

64. Thompson LR, Jon GSanders, McDonald D, Amir A, Ladau J, Locey KJ, et al. A communal catalogue reveals Earth's multiscale microbial diversity. Nature 2017;551:457-63.

65. Pésant S, Fabrice Not, Picheral M, Kandels-Lewis S, Bescot NL, Gorsky G, et al. Open science resources for the discovery and analysis of Tara Oceans data. Sci Data. 2015;2:150023.

66. Trembath-Reichert E, Case DH, Orphan VJ. Characterization of microbial associations with methanotrophic archaea and sulfatereducing bacteria through statistical comparison of nested Magneto-FISH enrichments. PeerJ. 2016;4:e1913 https://doi.org/ 10.7717/peerj.1913.

67. Teske A, McKay LJ, Ravelo AC, Aiello I, Mortera C, NúñezUseche F, et al. Characteristics and evolution of sill driven offaxis hydrothermalism in Guaymas Basin - the Ringvent site. Sci Rep. 2019;9:13847.

68. Trias R, Ménez B, Campion PL, Zivanovic Y, Lecourt L, Lecoeuvre A, et al. High reactivity of deep biota under anthropogenic $\mathrm{CO}_{2}$ injection into basalt. Nat Commun. 2017;8:1063.

69. Alfredsson HA, Oelkers EH, Hardarsson BS, Franzson H, Gunnlaugsson E, Gislason SR. The geology and water chemistry of the Hellisheidi, SW-Iceland carbon storage site. Int J Greenh Gas Control. 2013;12:399-418.

70. Matsushita M, Ishikawa S, Magara K, Sato Y, Kimura H. The potential for $\mathrm{CH}_{4}$ production by syntrophic microbial communities in diverse deep aquifers associated with an accretionary prism and its overlying sedimentary layers. Microbes Environ. 2020;35:1-10. https://doi.org/10.1264/jsme2.ME19103.

71. Matsushita M, Magara K, Sato Y, Shinzato N, Kimura H. Geochemical and microbiological evidence for microbial methane production in deep aquifers of the Cretaceous accretionary prism. Microbes Environ. 2018;33:205-13.

72. An D, Caffrey SM, Soh J, Agrawal A, Brown D, Budwill K, et al. Metagenomics of hydrocarbon resource environments indicates aerobic taxa and genes to be unexpectedly common. Environ Sci Technol. 2013;47:10708-17.

73. Bachu S. Basin-scale geology and hydrogeology of coalbed-methane bearing strata in Rocky Mountain sedimentary basins. (Alberta Research Council, Edmonton, AB, Canada, 1997).

74. Kjeldsen KU, Kjellerup BV, Egli K, Frølund B, Nielsen PH, Ingvorsen K. Phylogenetic and functional diversity of bacteria in biofilms from metal surfaces of an alkaline district heating system. FEMS Microbiol Ecol. 2007;61:384-97.

75. Magnabosco C, Tekere M, Lau MCY, Linage B, Kuloyo O, Erasmus M, et al. Comparisons of the composition and biogeographic distribution of the bacterial communities occupying South African thermal springs with those inhabiting deep subsurface fracture water. Front Microbiol. 2014;5:679-89.

76. Willerslev E, Hansen AJ, Rønn R, Brand TB, Barnes I, Wiuf C, et al. Long-term persistence of bacterial DNA. Curr Biol. 2004;14: R9-R10.

77. Liang R, Lau M, Baars O, Robb FT, Onstott TC. Aspartic acid racemization constrains long-term viability and longevity of endospores. FEMS Microb Ecol. 2019;95:fiz132.

78. Volpi M, Lomstein BA, Sichert A, Røy H, Jørgensen BB, Kjeldsen KU. Identity, abundance, and reactivation kinetics of thermophilic fermentative endospores in cold marine sediment and seawater. Front Microbiol. 2017;8:131.

79. Jungbluth SP, TGd Rio, Tringe SG, et al. Comparisons of a bacterial lineage that inhabits both marine and terrestrial deep subsurface systems. PeerJ 2017;5:e3134. 
80. Lawver LA, Coffin MF, Dalziel IWD, Gahagan LM, Campbell DA. The PLATES 2001 Atlas of Plate Reconstructions (750 Ma to Present Day). UTIG Technical Report No. 189. (The University of Texas Institute for Geophysics, Austin, Texas, 2001).

81. Torsvik TH, Amundsen HEF, Trønnes RG, Doubrovine PV, Gaina C, Kusznir NJ, et al. Continental crust beneath southeast Iceland. Proc Natl Acad Sci USA. 2015;112:E1818-E27.

82. Barnes GL. Origins of the Japanese Islands: the New "Big Picture". Jpn Rev. 2003;15:3-50.

83. Martinez-Gutierrez CA, Aylward FO. Strong purifying selection Is associated with genome streamlining in epipelagic Marinimicrobia. Genome Biol Evol. 2019;11:2887-94.

84. Hu J, Blanchard JL. Environmental sequence data from the Sargasso Sea reveal that the characteristics of genome reduction in Prochlorococcus are not a harbinger for an escalation in genetic drift. Mol Biol Evol. 2009;26:5-13.

85. Luo H, Swan BK, Stepanauskas R, Hughes AL, Moran MA. Comparing effective population sizes of dominant marine alphaproteobacteria lineages. Environ Microbiol Rep. 2014;6:167-72.

86. Shapiro BJ, Alm EJ. Comparing patterns of natural selection across species using selective signatures. Plos Genet. 2008;4:e23.
87. Chen X, Chen Z, Chen H, Su Z, Yang J, Lin F, et al. Nucleosomes suppress spontaneous mutations base-specifically in eukaryotes. Science 2012;335:1235-8.

88. Lujan SA, Clausen AR, Clark AB, MacAlpine HK, MacAlpine DM, Malc EP, et al. Heterogeneous polymerase fidelity and mismatch repair bias genome variation and composition. Genome Res. 2014;24:1751-64.

89. Park Y, Choi H, Lee DS, Kim Y. Improvement of the $3^{\prime}-5^{\prime}$ exonuclease activity of Taq DNA polymerase by protein engineering in the active site. Mol Cells. 1997;7:419-24.

90. Dean FB, Nelson JR, Giesler TL, Lasken RS. Rapid amplification of plasmid and phage DNA using phi29 DNA polymerase and multiply-primed rolling circle amplification. Genome Res. 2001;11:1095-9.

91. Konstantinidis KT, Rossello-Mora R, Amann R. Uncultivated microbes in need of their own taxonomy. ISME J. 2017;11:2399-406.

92. Parks DH, Chuvochina M, Waite DW, Rinke C, Skarshewski A, Chaumeil PA, et al. A standardized bacterial taxonomy based on genome phylogeny substantially revises the tree of life. Nat Biotechnol. 2018;36:996-1004. https://doi.org/10.1038/ nbt.4229. 